\title{
Field-Induced Deformation as a Mechanism for Scanning Tunneling Microscopy Based
} Nanofabrication

\author{
Hansen, Ole; Ravnkilde, Jan Tue; Quaade, Ulrich; Stokbro, Kurt; Grey, Francois
}

Published in:

Physical Review Letters

Link to article, DOI:

10.1103/PhysRevLett.81.5572

Publication date:

1998

Document Version

Publisher's PDF, also known as Version of record

Link back to DTU Orbit

Citation (APA):

Hansen, O., Ravnkilde, J. T., Quaade, U., Stokbro, K., \& Grey, F. (1998). Field-Induced Deformation as a

Mechanism for Scanning Tunneling Microscopy Based Nanofabrication. Physical Review Letters, 81(25), 5572 5575. https://doi.org/10.1103/PhysRevLett.81.5572

\section{General rights}

Copyright and moral rights for the publications made accessible in the public portal are retained by the authors and/or other copyright owners and it is a condition of accessing publications that users recognise and abide by the legal requirements associated with these rights.

- Users may download and print one copy of any publication from the public portal for the purpose of private study or research.

- You may not further distribute the material or use it for any profit-making activity or commercial gain

- You may freely distribute the URL identifying the publication in the public portal 


\title{
Field-Induced Deformation as a Mechanism for Scanning Tunneling Microscopy Based Nanofabrication
}

\author{
O. Hansen, J. T. Ravnkilde, U. Quaade, K. Stokbro, and F. Grey \\ Mikroelektronik Centret, Technical University of Denmark, Building 345e, DK-2800 Lyngby, Denmark
}

(Received 15 July 1998)

\begin{abstract}
The voltage between tip and sample in a scanning tunneling microscope (STM) results in a large electric field localized near the tip apex. The mechanical stress due to this field can cause appreciable deformation of both tip and sample on the scale of the tunnel gap. We derive an approximate analytical expression for this deformation and confirm the validity of the result by comparison with a finite element analysis. We derive the condition for a field-induced jump to contact of tip and sample and show that this agrees well with experimental results for material transfer between tip and sample by voltage pulsing in ultrahigh vacuum. [S0031-9007(98)08020-X]
\end{abstract}

PACS numbers: 61.16.Ch, 41.20.Cv, 74.25.Ld

In a scanning tunneling microscope (STM), the separation between the tip and sample is a key parameter for quantitative interpretation of the images and spectroscopic data that the STM produces. Normally, this distance is not accessible to direct measurement and must instead be inferred from measurements of current, voltage, and displacement of the piezoelectric scanner tube. Direct interaction due to the interatomic potentials between atoms on the tip and the sample can cause significant deformations of both tip and sample [1-6]. Such direct interaction has important consequences for measurements of the absolute tip position [7], the tunnel barrier height [8], the atomic corrugation of surfaces [1,9], and the phenomenon of jump to contact, where a mechanical instability causes tip and sample to suddenly join $[3,4]$.

In this Letter, we show how the electric field due to the applied bias between the tip and sample can also be a significant source of elastic deformation. This possibility has not received attention previously, because most experimental studies of tip-sample interaction attempt to minimize any field effects by using very low biases $[9,10]$, while in theoretical studies, field effects are usually neglected [5]. We find, however, that the field induced deformation of both tip and sample is significant at typical scanning voltages. Further, we determine the conditions under which this purely elastic deformation results in jump to contact and show that they agree quantitatively with published experimental results by Guo and Thompson [11] for material transfer between tip and sample by voltage pulsing. Field-induced elastic jump to contact is thus an alternative to the field evaporation mechanism proposed in many STM nanofabrication experiments [11-13] the validity of which remains controversial $[11,14]$.

The theoretical approach in this study is to develop a simple yet accurate analytical expression for the elastic deformation of tip and surface using classical continuum mechanics and electrostatics. The accuracy of the analytical expression is determined by comparison with a finite element analysis of tip and sample modeled as continuous elastic media. This approach does not give detailed atomic-scale information, but it has the advantage that it can be applied to systems much larger than those accessible to molecular dynamics simulations.

As shown in the inset of Fig. 1, we treat the tip to a first approximation as a spherical cap of radius $R$ on a truncated cone with the half opening angle $\theta_{0}$. With no applied field, the tunnel gap is $h_{0}$ and with the field induced deformation it is $h$. Typical values in STM are $h=5 \AA, R=1000 \AA$ [15]; in other words, $h \ll R$. We assume cylindrical symmetry, with $r$ the radial coordinate and $z$ the vertical coordinate. The sample surface is the plane defined by $z_{s}(r)=0$. The tip surface near the tip apex is $z_{t}(r)=h+R(1-\cos \varphi)$, where $\varphi=\arcsin \left(\frac{r}{R}\right)$ is the angle measured from the center of the spherical cap of the tip (see Fig. 1 inset).

The exact electric field $E_{t}$ and $E_{s}$ on the tip and sample (both assumed metallic) cannot be expressed in closed form [16]. However, simple yet accurate expressions for the electric field near the apex can be derived from a concentric spheres model [17]

$$
\begin{gathered}
E_{t}(\varphi) \simeq \frac{V}{h+R[1-\cos (\varphi)]}\left(1+\frac{h}{R}\right), \\
E_{S}(r) \simeq \frac{V}{\left[\sqrt{r^{2}+(R+h)^{2}}-R\right]} \frac{R}{\sqrt{r^{2}+(R+h)^{2}}} .
\end{gathered}
$$

To a first approximation the electric field on the tip or the sample falls to half its maximum value at a characteristic radius $r_{c} \simeq \sqrt{2 R h} \ll R$.

The electrostatic pressure on a metallic surface due to the electric field $E$ on the surface is $p(r)=\frac{1}{2} \varepsilon E^{2}(r)$ [18], where $\varepsilon$ is the permittivity of the dielectric between the tip and sample surfaces. The deformation of the tip and the sample due to the electrostatic pressure can be calculated from elastic theory using a superposition of Boussinesq's solution to a point force load on a semi-infinite sample [19]. The deformation $w_{s}(r)$ of the sample in the $z$ direction becomes 


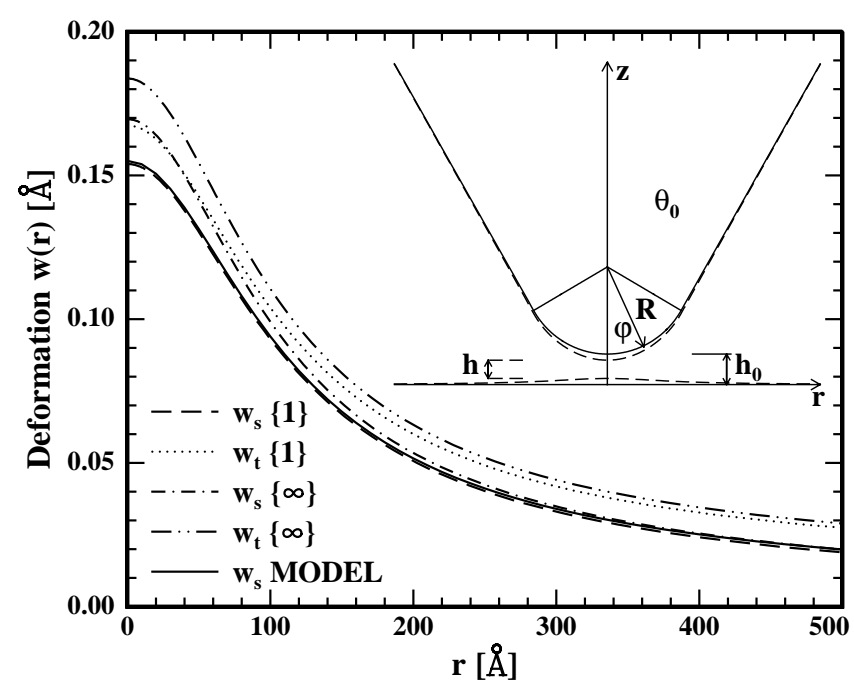

FIG. 1. The deformation of a tungsten sample $w_{s}(r)$ and tip $w_{t}(r)$ as a function of the radial position from ANSYS finite element calculations; both the converged results $\left(w_{s, t}\{\infty\}\right)$ and results from the first iteration $\left(w_{s, t}\{1\}\right)$ are shown. For comparison also, the sample deformations calculated from the model Eq. (3) (using $h=h_{0}$ ) are shown. The applied bias is $V=5 \mathrm{~V}$. The inset shows a schematic of the STM tip and sample: $R$ is the radius of the spherical cap, $h$ and $h_{0}$ the deformed and relaxed tip to sample distance, respectively, $\theta_{0}$ is the half top angle of the truncated conical tip shank, and $\varphi$ is the polar angle. The deformed surfaces are indicated with dashed lines.

$$
w_{s}(r)=\frac{\left(1-\nu_{s}^{2}\right)}{\pi Y_{s}} \int_{0}^{2 \pi} \int_{0}^{\infty} p_{s}\left(r^{\prime}\right) d \lambda d \phi,
$$

with $\left(r^{\prime}\right)^{2}=r^{2}+\lambda^{2}-2 r \lambda \cos \phi . p_{s}$ is the pressure on the sample, $Y_{s}$ is Young's modulus, and $\nu_{s}$ is Poisson's ratio of the sample. At the apex the angular integral is trivial and the deformation $w_{s 0}=w_{s}(0)$ becomes

$$
\begin{aligned}
w_{s 0} & =\frac{2\left(1-\nu_{s}^{2}\right)}{Y_{s}} \int_{0}^{\infty} p_{s}(r) d r \\
& \simeq \frac{\left(1-\nu_{s}^{2}\right)}{Y_{s}} p_{s 0} \frac{\pi}{2} \sqrt{2 R h}
\end{aligned}
$$

where $p_{s 0}=p_{s}(0)$.

Below, we shall show by comparison with ANSYS [20] finite element analysis that Eq. (3) accurately reproduces the deformation of the sample. A typical geometry used in finite element analysis is $R=1000 \AA$ and $h=5 \AA$ (the units are in principle arbitrary in this simulation, since tip and sample are treated as continuous media). The spherical tip apex is joined smoothly with a truncated cone of half opening angle $30^{\circ}$ (angles in the range $20^{\circ}-40^{\circ}$ give almost identical results). The tip extends to $z=10000 \AA$ and the sample to $z=-10000 \AA$ and $r=9525 \AA$. These boundaries are effectively frozen during calculations of elastic relaxation. An adaptive meshing and elements with curved surfaces are used. In the calculations the smallest node separation is $0.5 \AA$ (near the tip apex). Calculations on tips with a small hemi- spherical protrusion at the apex have also been performed [21] and give results similar to those presented here.

An iterative procedure is carried out where the tip and sample surfaces are fixed and the electric field is calculated; the stress due to this field is then fixed and the elastic relaxation of tip and sample is calculated. The deformed tip is used in a new calculation of the electric field followed by a new calculation of the stress and elastic relaxation. For sufficiently small $V$, this calculation converges at the first iteration and is well approximated by Eq. (3) using $h=h_{0}$. In Fig. 1 the deformations of the tip and sample surfaces are shown as a function of the radial distance from apex obtained from a converged iterative finite element calculation. The elastic parameters used are those of tungsten $(Y=$ $411 \mathrm{GPa}, \nu=0.28$ [22]). For comparison, the sample deformation calculated from Eq. (3) is shown.

From Fig. 1 we see that the deformation of the tip is very similar to the deformation of the sample. The reason is that as long as $R \gg h$, the electric field and resulting stress are confined to a region $r_{c} \simeq \sqrt{2 R h} \ll R$. On this scale tip and sample behave roughly symmetrically, so $w_{s}(r) \approx w_{t}(r)$. Hence, the deformation $w_{t 0}$ of the tip at the apex is approximately

$$
w_{t 0} \simeq \frac{\left(1-\nu_{t}^{2}\right)}{Y_{t}} p_{t 0} \frac{\pi}{2} \sqrt{2 R h},
$$

where $Y_{t}$ is Young's modulus and $\nu_{t}$ is Poisson's ratio of the tip material. $p_{t 0}$ is the tip apex pressure. Thus, the reduction in tunneling distance $w_{0}=w_{s 0}+w_{t 0}$ becomes

$$
w_{0} \simeq \frac{p_{0}}{Y_{r}} \frac{\pi}{2} \sqrt{2 R h},
$$

where $Y_{r}$ is the reduced Young's modulus for the tipsample system $\frac{1}{Y_{r}}=\frac{\left(1-\nu_{t}^{2}\right)}{Y_{t}}+\frac{\left(1-\nu_{s}^{2}\right)}{Y_{s}}$ and $p_{0}=\frac{1}{2} \varepsilon\left(\frac{V}{h}\right)^{2} \simeq$ $p_{s 0} \simeq p_{t 0}$.

At a sufficiently large bias voltage $V_{C}$, the system becomes unstable and the tip and sample jump to contact. The jump-to-contact voltage can be estimated from an investigation of the derivative of the elastic and the electrostatic forces in equilibrium or by solving Eq. (6) for the applied voltage needed to achieve a given reduction in tunnel distance

$$
V\left(w_{0}\right)=\sqrt{\frac{4 Y_{r}}{\varepsilon \pi} \frac{w_{0}\left(h_{0}-w_{0}\right)^{2}}{\sqrt{2 R\left(h_{0}-w_{0}\right)}} .}
$$

The jump-to-contact condition is then $\frac{\partial V\left(w_{0}\right)}{\partial w_{0}}=0$, which is fulfilled at $w_{C}=\frac{2}{5} h_{0}$. Hence, at jump to contact the tunnel gap is $h_{C}=\frac{3}{5} h_{0}$. From Eq. (7) the jump-to-contact voltage $V_{C}$ is obtained

$$
V_{C}=\sqrt{\frac{24}{25} \sqrt{\frac{3}{5}}} \cdot \sqrt{\frac{Y_{r}}{\varepsilon \pi} \frac{h_{0}^{3}}{\sqrt{2 R h_{0}}}} .
$$

The deformation of the sample surface, however, leads to a finite radius of curvature $\rho_{s}$ for the sample surface near apex. From Eq. (3) the sample radius of curvature $\rho_{s}=$ $\frac{4}{3} R h_{0} / w_{s 0}$ is obtained. Detailed calculations [21] show 
that a similar correction applies to the curvature of the tip. It follows that the sample radius of curvature is comparable to the tip radius $R$ when the sample deformation $w_{s 0}$ is comparable to the initial tip-to-sample distance $h_{0}$. Hence, an effective radius $R_{\text {eff }}$ of the tip-sample system must be used in the calculations

$$
\frac{1}{R_{\mathrm{eff}}}=\frac{1}{R}+\frac{3}{4} \frac{w_{t 0}}{R h_{0}}+\frac{3}{4} \frac{w_{s 0}}{R h_{0}}=\frac{1}{R}\left(1+\frac{3}{4} \frac{w_{0}}{h_{0}}\right) .
$$

Using the effective radius of curvature $R_{\text {eff }}$ in Eq. (7) yields a corrected applied voltage $V_{\rho}\left(w_{0}\right)$ to sustain a given reduction in the tunnel gap

$$
V_{\rho}\left(w_{0}\right)=\sqrt{\frac{4 Y_{r}}{\varepsilon \pi} \frac{w_{0}\left(h_{0}-w_{0}\right)^{2}}{\sqrt{2 R\left(h_{0}-w_{0}\right)}} \sqrt{1+\frac{3}{4} \frac{w_{0}}{h_{0}}}} .
$$

In this case the jump to contact condition is fulfilled for a reduction in tunneling gap of $w_{C}=$ $(\sqrt{697}-11) / 36 h_{0} \simeq 0.428 h_{0} . \quad$ Simultaneously, the jump-to-contact voltage is increased by $7 \%$ compared to the value predicted from Eq. (8). The jump-to-contact voltage $V_{C}$ is seen to be rather insensitive to the radius of curvature of the tip $\left(V_{C} \propto R^{-0.25}\right)$, quite sensitive to the reduced Young's modulus $\left(V_{C} \propto \sqrt{Y_{r}}\right)$, and very sensitive to the equilibrium tunnel gap $\left(V_{C} \propto h_{0}^{1.25}\right)$.

In Fig. 2 the tunnel gap calculated from Eqs. (7) and (10) as a function of the applied bias voltage is compared to finite element calculations. The model calculations and the finite element calculations are seen to be in excellent agreement. Close to the jump-to-contact condition, the radius of curvature correction is seen to improve the model predictions.

Measurements of the threshold voltage for pulsed voltage material transfer between tip and sample as a function of the low bias tunnel resistance [11] are reproduced in Fig. 3 for a tungsten tip and gold sample. The jump-tocontact voltage calculated using Eq. (7) with the elastic parameters for $\mathrm{Au}(Y=78.5 \mathrm{GPa}, \nu=0.42$ [22] $)$ and $\mathrm{W}$ [23], is shown in the same figure, with $h_{0}$ determined from the measured tunnel resistance $R_{T}$ using [24]

$$
\frac{1}{R_{T}}=\frac{e^{2}}{2 \pi \hbar} \frac{3 A_{T}}{4 \pi h} \sqrt{\frac{2 m \Phi}{\hbar^{2}}} \exp \left(-2 h \sqrt{\frac{2 m \Phi}{\hbar^{2}}}\right),
$$

where $\Phi$ (here $4.5 \mathrm{eV}$ ) is the barrier height, $e$ is the unit charge, $m$ the electron mass, and $\hbar$ Planck's constant. Assuming a tip radius of $R=1000 \AA$, the only unknown parameter is the tunnel current cross section, $A_{T}$. A value in the range $A_{T}=3 \AA^{2}$ (solid line) to $A_{T}=6 \AA^{2}$ (dashed line), corresponding to tunneling from a single atom on the tip, agrees well with the measurements. Thus we find that the measurements are fully consistent with a fieldinduced elastic jump to contact between tip and sample. It follows that we would expect an ordering of voltage thresholds according to the square root of the reduced Young's modulus of the tip-sample system if material transfer experiments are performed at a fixed initial tip to

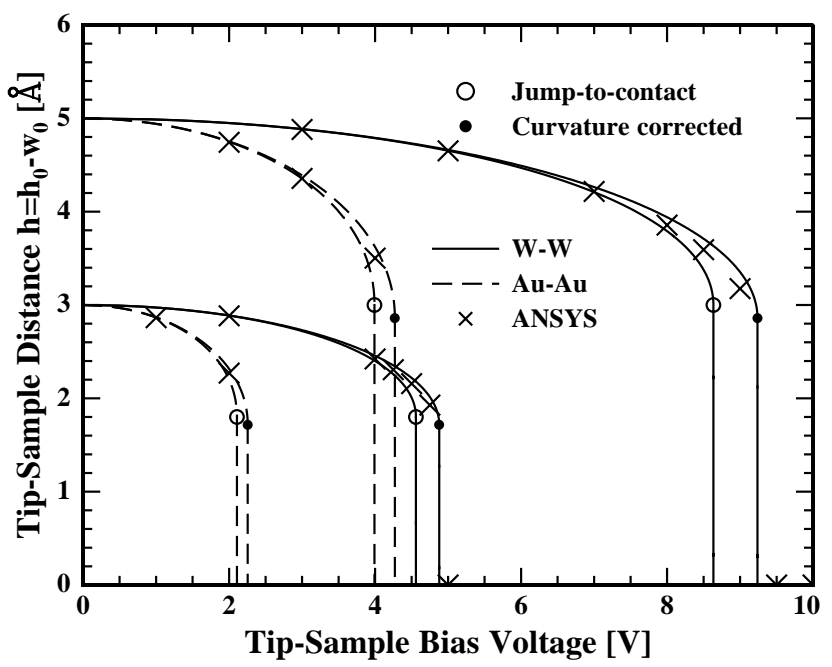

FIG. 2. The tunnel gap as a function of the applied bias voltage. The full lines show the calculated tunnel gap for the case of a W tip and sample, whereas the dashed lines are for an Au tip and sample. In both cases calculations are shown for a field-free tunnel distance of 3 and $5 \AA$. The crosses are data from ANSYS finite element simulations. Calculations with and without radius of curvature correction are shown.

sample separation $h_{0}$. Other measurements of threshold voltage for material transfer exist [12], but these are in air, where the situation is considerably more complex, due to the presence of water.

The surface strain $\epsilon_{z}$ normal to the surface due to the electrostatic pressure is $\epsilon_{z}=\left(1-\nu-2 \nu^{2}\right) \frac{p}{Y}$ [19]. However, the maximum on-axis strain in the same direction is found below the surface and is roughly $25 \%$ higher (dependent on Poisson's ratio) than the surface on-axis

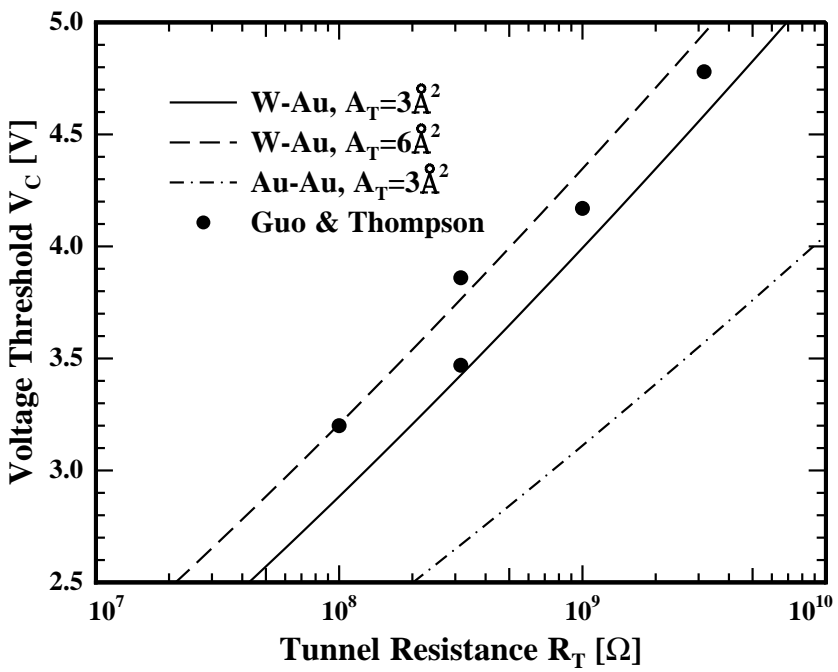

FIG. 3. Voltage threshold for material transfer between a gold sample and a tungsten tip as a function of the low bias voltage tunnel gap resistance, reproduced from Guo and Thompson [11]. Also shown is the calculated jump-to-contact voltage assuming a tip radius $R=1000 \AA$ and a tunnel current cross section $A_{T}=3 \AA^{2}$ (full line) or $6 \AA^{2}$ (dashed line). For comparison, a calculation for a gold tip with $A_{T}=3 \AA^{2}$ is shown (dash-dotted line). 
strain. At the edge of jump to contact the surface strain is obtained from $V_{C}$ Eq. (8):

$$
\begin{aligned}
\epsilon_{z c} & =\frac{\left(1-\nu-2 \nu^{2}\right) \varepsilon}{2 Y} \frac{V_{C}^{2}}{h_{c}^{2}} \\
& =\frac{Y_{r}}{Y} \frac{4\left(1-\nu-2 \nu^{2}\right)}{\pi \sqrt{15}} \sqrt{\frac{h_{0}}{2 R}},
\end{aligned}
$$

where $Y$ and $\nu$ are the elastic properties of the surface. We note that the use of expressions for a static system is justified because we estimate that the elastic deformations described here have response times in the picosecond range [25], whereas the shortest pulses used for pulse modification by STM are longer than $1 \mathrm{~ns}$.

If the tip and the sample are of the same material the surface strain at jump to contact becomes $\epsilon_{z c} \simeq 0.5 \%$ if $R=$ $1000 \AA$ and $h_{0}=5 \AA$, whereas a considerably sharper tip with $R=100 \AA$ has a jump-to-contact surface strain of $\epsilon_{z c} \simeq 1.5 \%$. As a result, jump to contact due to an electric field can be achieved by accumulation of small, elastic changes in local interatomic distances over a large volume. This contrasts with the large changes of interatomic distances $(>10 \%)$ observed in molecular dynamics simulations for jump to contact due to the interaction potential between tip and sample [4]. This discrepancy may occur in part because large-scale elastic deformation is precluded in molecular dynamics simulations, due to the small volume in which atomic coordinates are allowed to relax. Indeed, if jump to contact can be achieved by purely elastic deformation, then this will preempt mechanisms that have been proposed based on plastic deformation $[11,14,26]$.

In conclusion, the electric field $E$ between the tip of an STM and a surface produces an elastic extension of both tip and sample which reaches a value of about $\frac{1}{5} h_{0}$ before jump to contact occurs. Pulsed voltage experiments in vacuum are in quantitative agreement with field-induced elastic jump to contact as a mechanism for nanofabrication.

U. Q. and K.S. acknowledge support from the Danish Research Councils (STVF No. 9800466).

[1] J. M. Soler, A. M. Baro, N. Garcia, and H. Rohrer, Phys. Rev. Lett. 57, 444 (1986).

[2] J. B. Pethica, Phys. Rev. Lett. 57, 3235 (1986).

[3] J. B. Pethica and A.P. Sutton, J. Vac. Sci. Technol. A 6, 2490 (1988).

[4] U. Landman and W. D. Luedtke, J. Vac. Sci. Technol. B 9, 414 (1991).

[5] K. Cho and J. D. Joannopoulos, Phys. Rev. Lett. 71, 1387 (1993).

[6] U. Dürig, J. K. Gimzewski, and D. W. Pohl, Phys. Rev. Lett. 57, 2403 (1986).

[7] J. H. Coombs and J. B. Pethica, IBM J. Res. Dev. 30, 455 (1986).

[8] L. Olesen, M. Brandbyge, M. R. Sørensen, K. W. Jacobsen, E. Lægsgaard, I. Stensgaard, and F. Besenbacher, Phys. Rev. Lett. 76, 1485 (1996).

[9] A. R. H . Clarke, J. B. Pethica, J. A. Nieminen, F. Besenbacher, E. Lægsgaard, and I. Stensgaard, Phys. Rev. Lett.
76, 1276 (1996).

[10] U. Dürig, O. Züger, and D. W. Pohl, Phys. Rev. Lett. 65, 349 (1990).

[11] C.X. Guo and D. J. Thompson, Ultramicroscopy 42-44, 1452 (1992).

[12] H. J. Mamin, P. H. Guether, and D. Rugar, Phys. Rev. Lett. 65, 2418 (1990); C. S. Chang, W. B. Su, and T. T. Tsong, Phys. Rev. Lett. 72, 574 (1994); S. E. McBride and G. C. Wetsel, Appl. Phys. Lett. 59, 3056 (1991).

[13] J. Rabe and S. Buchholz, Appl. Phys. Lett. 58, 702 (1991); I.-W. Lyo and P. Avouris, Science 256, 173 (1991); H. Uchida, D. Huang, F. Grey, and M. Aono, Phys. Rev. Lett. 70, 2040 (1993).

[14] T.C. Chang, C.S. Chang, H. N. Lin, and T.T. Tsong, Appl. Phys. Lett. 67, 903 (1995).

[15] R. Zhang and D. G. Ivey, J. Vac. Sci. Technol. B 14, 1 (1996).

[16] S. Hudlet, M. Saint Jean, C. Guthmann, and J. Berger, Europhys. Lett. B 2, 5 (1998).

[17] A fixed inner sphere completing the spherical tip cap and a concentric outer sphere with a radius (variable) extending to the sample through the point where the field is to be determined.

[18] E. C. Jordan and K. G. Balmain, Electromagnetic Waves and Radiating Systems (Prentice-Hall, Englewood Cliffs, NJ, 1968), 2nd ed.

[19] S. P. Timoshenko and J. N. Goodier, Theory of Elasticity (McGraw-Hill, Singapore, 1982), 3rd ed.

[20] P. Kohnke, ANSYS Users Manual (ANSYS Inc., Canonsburg, 1996).

[21] O. Hansen, J. Tue Ravnkilde, U. Quaade, K. Stokbro, and F. Grey (to be published).

[22] E. A. Brandes and G. B. Brook, Smithells Metals Reference Book (Butterworth-Heinemann Ltd., Oxford, 1992), 7 th ed.

[23] Although some gold may absorb on the tip during pulsing, only a very thick coating on the tip would make it necessary to use the elastic parameters of gold in the calculation of the tip deformation.

[24] J. G. Simmons, J. Appl. Phys. 32, 1793 (1963).

[25] The elastic energy per unit area near the tip apex is $\quad \mathcal{E}_{\mathrm{el}}=\frac{1}{2} p_{s 0} w_{s 0}=\frac{1}{2} Y_{s} w_{s 0}^{2} /\left[\left(1-\nu_{s}^{2}\right) \frac{\pi}{2} \sqrt{2 R h}\right] \quad$ and the average kinetic energy per unit area due to a harmonic deformation at the frequency $\omega$ is $\mathcal{E}_{\text {kin }}=$ $\frac{1}{2} \int \varrho_{s}[\omega w(0, z)]^{2} d z \simeq \frac{1}{2} \varrho_{s} \omega^{2} w_{s 0}^{2} 1.5 \sqrt{2 R h}$. A characteristic time $\tau$ for the elastic deformation can be estimated as the reciprocal of the resonant frequency $\omega_{0}$ of a simple harmonic oscillator with these elastic and kinetic energies; hence $\tau \simeq \frac{1}{\omega_{0}}=$ $\sqrt{1.5 \pi\left(1-\nu_{s}^{2}\right) \varrho_{s} /\left[2 Y_{s}\right]} \sqrt{2 R h} \simeq 3$ ps in the case of a tungsten sample with mass density $\varrho_{s}=19.3 \mathrm{~g} / \mathrm{cm}^{3}$. Essentially the same result is obtained from an acoustic wave point of view; a sound wave with spherical symmetry would propagate in the sample at a velocity $\quad c_{l}=\sqrt{Y_{s}\left(1-\nu_{s}\right) /\left[\left(1+\nu_{s}\right)\left(1-2 \nu_{s}\right) \varrho_{s}\right]}$; hence, the response time $\tau$ of the system is estimated as the ratio of the characteristic dimension of the system to the sound velocity: $\tau \simeq \sqrt{2 R h} / c_{l}=$ $\sqrt{\left(1+\nu_{s}\right)\left(1-2 \nu_{s}\right) \varrho_{s} /\left[Y_{s}\left(1-\nu_{s}\right)\right]} \sqrt{2 R h} \simeq 2$ ps.

[26] J. I. Pasqual, J. Méndez, J. Gómez-Herrero, A. Baró, N. García, and V. T. Binh, Phys. Rev. Lett. 71, 1852 (1993). 